\title{
Spontaneous and stimulated Raman scattering in planar silicon waveguides
}

Wang, Sha, Meister, Stefan, Mahdi, Shaimaa, Franke, Bülent, Al-Saadi, Aws, et al.

Sha Wang, Stefan Meister, Shaimaa Mahdi, Bülent A. Franke, Aws Al-Saadi, Lars Zimmermann, Harald H. Richter, David Stolarek, Viktor Lisinetskii, Viachaslau Ksianzou, Sigurd K. Schrader, H. J. Eichler, "Spontaneous and stimulated Raman scattering in planar silicon waveguides," Proc. SPIE 7943, Silicon Photonics VI, 794315 (17 January 2011); doi: 10.1117/12.875504

SPIE. Event: SPIE OPTO, 2011, San Francisco, California, United States 


\title{
Spontaneous and stimulated Raman scattering in planar silicon waveguides
}

\author{
Sha Wang ${ }^{\mathrm{a}}$, Stefan Meister ${ }^{\mathrm{a}}$, Shaimaa Mahdi ${ }^{\mathrm{a}}$, Bülent A. Franke ${ }^{\mathrm{a}}$, Aws Al-Saadi ${ }^{\mathrm{a}}$, Lars \\ Zimmermann $^{\mathrm{b}}$, Harald H. Richter ${ }^{\mathrm{b}}$, David Stolarek ${ }^{\mathrm{b}}$, Viktor Lisinetskii ${ }^{\mathrm{c}}$, Viachaslau \\ Ksianzou $^{\mathrm{c}}$, Sigurd Schrader ${ }^{\mathrm{c}}$, H. J. Eichler ${ }^{\mathrm{a}}$
}

a Technische Universität Berlin, Institut für Optik und Atomare Physik, ER1-1, Str. des 17. Juni 135, 10623 Berlin, Germany

b Innovations for High Performance microelectronics (IHP) GmbH, Im Technologiepark 25, 15236 Frankfurt (Oder), Germany

c University of Applied Sciences Wildau, Engineering Physics, Bahnhofstrasse, 15745 Wildau, Germany,

\begin{abstract}
:
Raman scattering in planar silicon on insulator (SOI) waveguides with $2 \mu \mathrm{m}$ width, $220 \mathrm{~nm}$ height and $2 \mathrm{~cm}$ length is investigated. A cw Nd:YAP laser at $1340.6 \mathrm{~nm}$ with $7 \mathrm{GHz}$ FWHM spectral width is used as the pump source. A lensed fiber of $2.5 \mu \mathrm{m}$ focus diameter is used to couple the pump laser into the waveguide. The coupling efficiency is estimated to be around 10\%. Spontaneous Raman scattering is observed with as low as $2.5 \mathrm{~mW}$ pump power inside the waveguide. The spontaneous Raman spectrum is measured by an optical spectrum analyzer. The first order Raman peak is measured at around $1441.4 \mathrm{~nm}$ corresponding to a Raman shift of $15.6 \mathrm{THz}$, while the FWHM of Raman spectrum is measured as around $100 \mathrm{GHz}$. Maximum Raman output of around $90 \mathrm{pW}$ is obtained by around 22 $\mathrm{mW}$ pump. The stimulated Raman gain coefficient is estimated as around $56 \mathrm{~cm} / \mathrm{GW}$ from the relationship between spontaneous Raman output power and pump power. A temperature dependence of Raman frequency shift of about $0.6 \mathrm{GHz} / \mathrm{K}$ is measured. The spontaneous anti-Stokes Raman scattering output peak at $1253 \mathrm{~nm}$ is also observed with around $35 \mathrm{~mW}$ pump. Stimulated Raman amplification measurement is carried out with a SLED white light source as probe signal. With $35 \mathrm{~mW}$ pump power, around $0.6 \mathrm{~dB}$ gain has been determined with both pump and probe being TE polarized.
\end{abstract}

\section{INTRODUCTION}

Silicon photonics is one of the most hotly research areas these days. Silicon-On-Insulator is believed to be the platform for next generation electronic Integrated Circuits. The silicon Integrated Circuits manufacturing is well developed. Silicon wafers have the lowest cost per unit area and the highest

Silicon Photonics VI, edited by Joel A. Kubby, Graham T. Reed, Proc. of SPIE Vol. 7943,

$794315 \cdot$ ? 2011 SPIE · CCC code: $0277-786 X / 11 / \$ 18 \cdot$ doi: 10.1117/12.875504

Proc. of SPIE Vol. $7943794315-1$ 
crystal quality compared with other semiconductor materials. The availability of high quality SOI wafers is an ideal platform for creating planar waveguide circuits. The strong optical confinement offered by the high index contrast between silicon $(n \approx 3.5)$ and $\mathrm{SiO}_{2}(\mathrm{n} \approx 1.5)$ makes it possible to scale photonic devices to several hundreds of nanometer level. Submicron-sized dimension will enhance the nonlinear intensity in the devices; it is easier to observe nonlinear optical phenomena, such as Raman and Kerr effects, in the chip-scale devices.

In this paper, Raman effect in planar silicon on insulator (SOI) waveguides with $2 \mu$ m width, $220 \mathrm{~nm}$ height and $2 \mathrm{~cm}$ length is investigated.

\section{SPONTANEOUS RAMAN EMISSION IN PLANAR SILICON}

In silicon the strongest Stokes peak corresponds to first order Raman scattering from the three fold degenerate optical modes at the center of the Brillouin zone [1]. It lies at $15.6 \mathrm{THz}$ away from the pump and with a FWHM of $105 \mathrm{GHz}$ at room temperature [2].

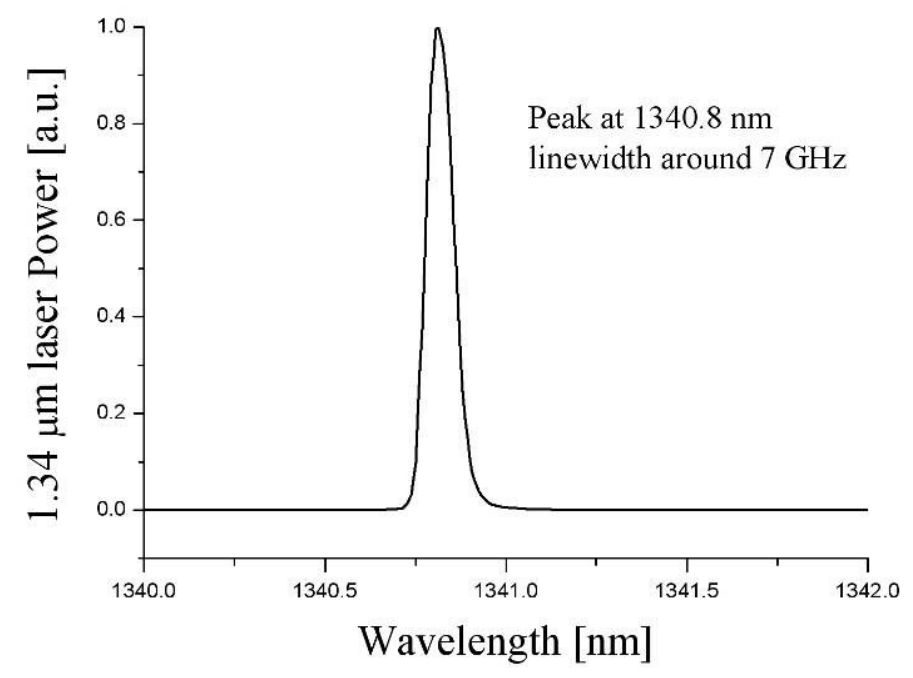

Fig.1 Spectrum of Nd:YAP laser at $1341 \mathrm{~nm}$

In order to observe the Raman emission in silicon waveguide, a LD end pumped cw Nd:YAP laser working at $1341 \mathrm{~nm}$ is used as the pump source. The silicon waveguide is with $2 \mu \mathrm{m}$ width, $220 \mathrm{~nm}$ height and $2 \mathrm{~cm}$ length. The spectrum of pump laser is shown in Fig.1. The pump laser is peak at $1340.8 \mathrm{~nm}$ has a bandwidth of around $7 \mathrm{GHz}$ detected from the OSA. Maximum pump power of 350 $\mathrm{mW}$ at $1341 \mathrm{~nm}$ is achievable. A half-wave plate in combination with a polarizer are used together to change the input pump power to silicon waveguide. A fiber polarization controller is used to change the polarization of pump laser in the silicon waveguide. The experimental setup is shown in Fig. 2. The 
$1341 \mathrm{~nm}$ pump is coupled into the waveguide through a single mode tapered fiber. The $2 \mu \mathrm{m} \times 220 \mathrm{~nm}$ silicon waveguide comprises a $5 \mu \mathrm{m} \times 220 \mathrm{~nm}$ tapered waveguide in order to decrease the coupling loss from fiber to silicon waveguide. The coupling loss is estimated to around $10 \mathrm{~dB}$ from our previous experiments [3]. Spontaneous Raman scattering at around $1441.6 \mathrm{~nm}$ is generated, which corresponds to a silicon Raman shift of $15.64 \mathrm{THz}$. The measured Raman spectrum is shown in Fig.3. The figure shows both the wavelength and the frequency shift of the Raman output.

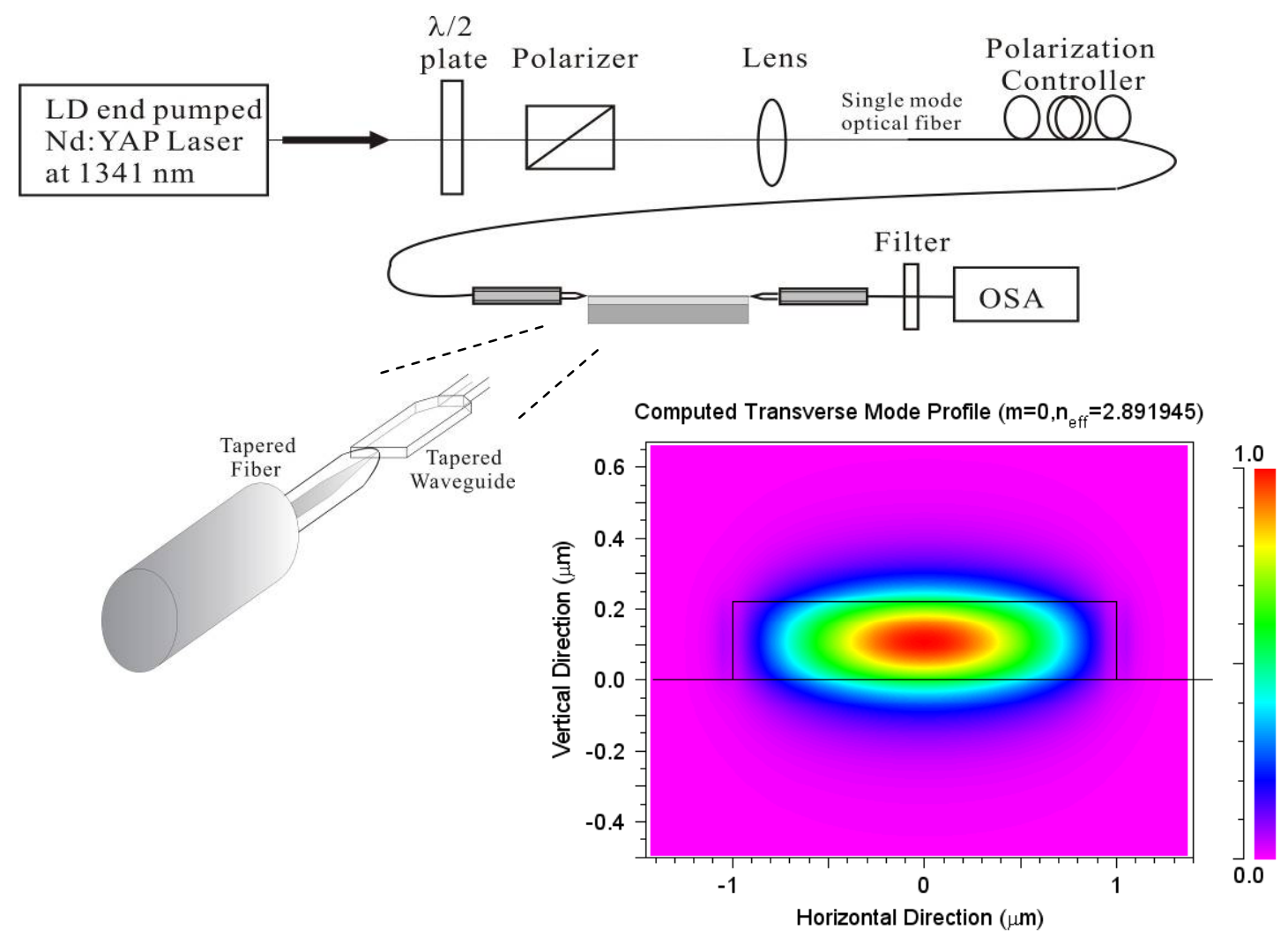

Fig.2 Experimental setup of Raman scattering measurement in silicon waveguide 


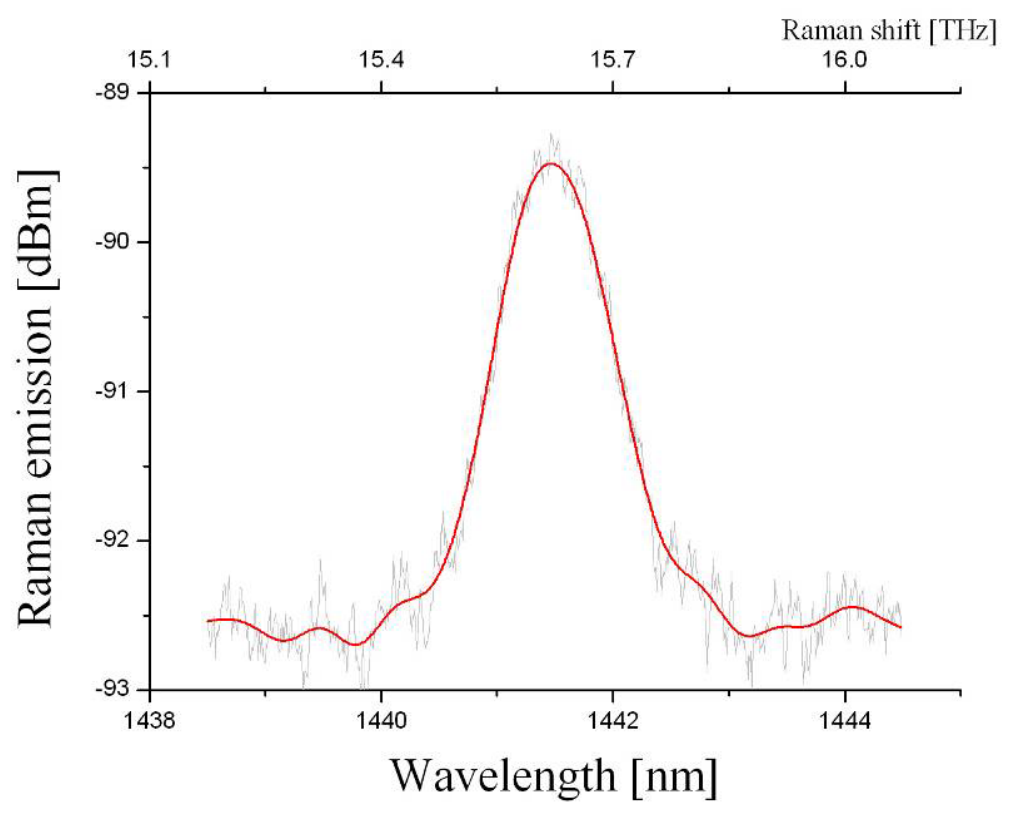

Fig.3 Raman spectrum of silicon waveguide

The forward Raman signal and the pump beam are then coupled into a receiving tapered fiber and are separated by a long pass filter. The signal is recorded by an optical spectrum analyzer (OSA) with 0.5 $\mathrm{nm}$ resolution. In the right down part of Fig. 2 is the calculated mode profile in $2 \mu \mathrm{m}$ silicon waveguide. The effective core area of the waveguide is defined as [4]

$A_{e f f}=\frac{\left[\iint I(x, y) d x d y\right]^{2}}{\iint I^{2}(x, y) d x d y}$

where $\mathrm{I}(\mathrm{x}, \mathrm{y})$ is the transverse intensity profile of the fundamental mode of the waveguide. From the calculated mode profile, the effective core area is estimated as around $0.68 \mu \mathrm{m}^{2}$.

Fig.4 shows the different results of two different pump polarization directions: TE pump and TM pump. The Raman shifts of both TE and TM pump are the same, which is measured as $15.64 \mathrm{THz}$. 


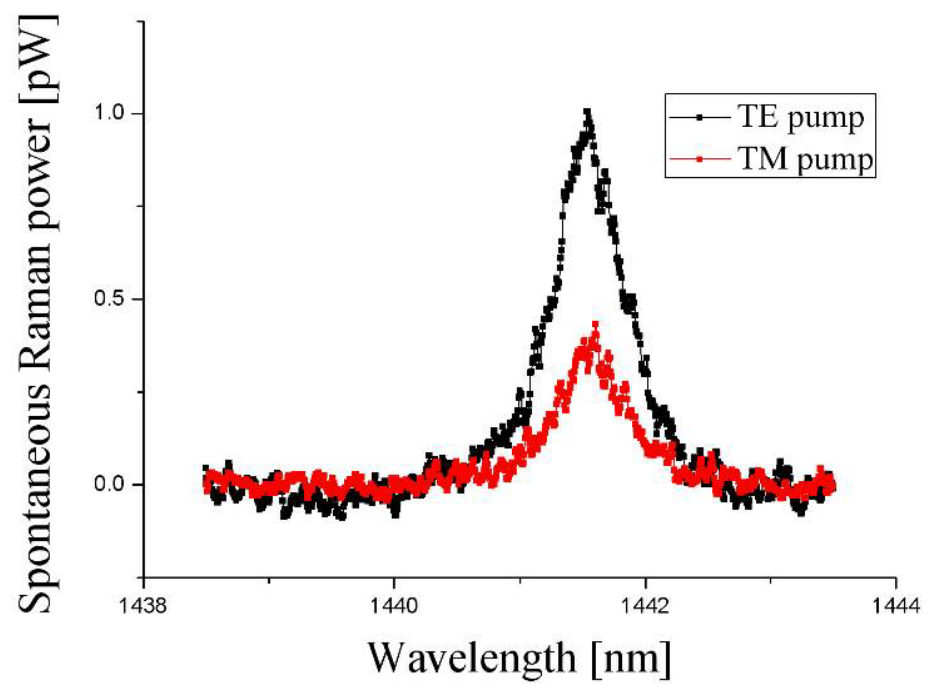

Fig.4 Spontaneous Raman spectra with TE and TM pump

Due to the much smaller bandwidth of pump laser compared with Raman gain bandwidth, the bandwidth of measured spontaneous Raman emission is near the same with the Raman linewidth of silicon. The output Spontaneous Raman bandwidths of both TE and TM pump are measured as around $100 \mathrm{GHz}$ (note: $100 \mathrm{GHz}$ are about $0.8 \mathrm{~nm}$-> so the OSA resolution should be as low as $0.2 \mathrm{~nm}$ ), which is within error compared with reported value $105 \mathrm{GHz}$. The power of spontaneous Raman emission with TE pump is around 3 times larger than with TM pump. This is because that the propagation loss of TE mode is smaller than of TM mode in such a small size silicon waveguide.

Fig. 5 gives the spontaneous Raman spectra at various TE pump power. As low as $25 \mathrm{~mW}$ pump power, i.e. less than $2.5 \mathrm{~mW}$ pump power in silicon waveguide, the efficient Raman conversion is observed. This is because of the small bandwidth of our pump laser, as well as the small effective mode area within the silicon waveguide. The spontaneous Raman output power is linear dependent with pump power.

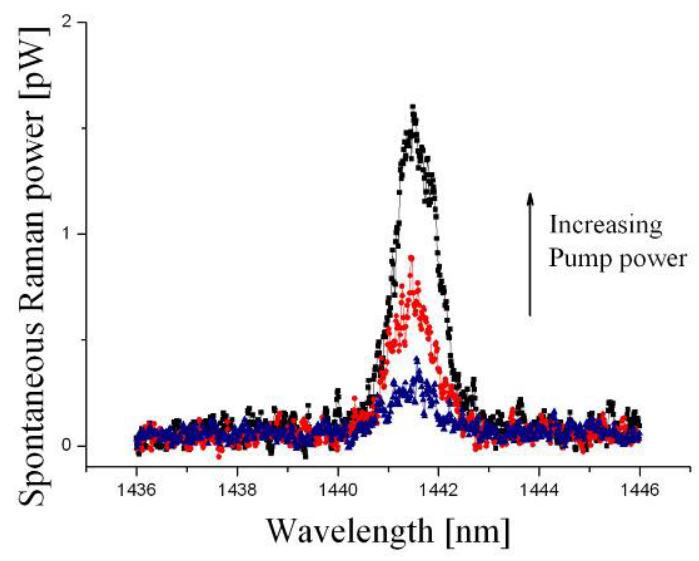


Fig. 5 spontaneous Raman spectra at various TE pump power

The temperature dependent Raman frequency shift is also studied. A TEC temperature controller is used to change the temperature of the waveguide from $290 \mathrm{~K}$ to $330 \mathrm{~K}$. Here the absorption induced temperature rising is not considered, because the thermal conductivity of silicon at around $300 \mathrm{~K}$ is reported larger than $100 \mathrm{Wm}^{-1} \mathrm{~K}^{-1}$ [5]. The pump is TE polarized and the power is not changed during measurement at different temperatures. Fig.6 shows the measured results.

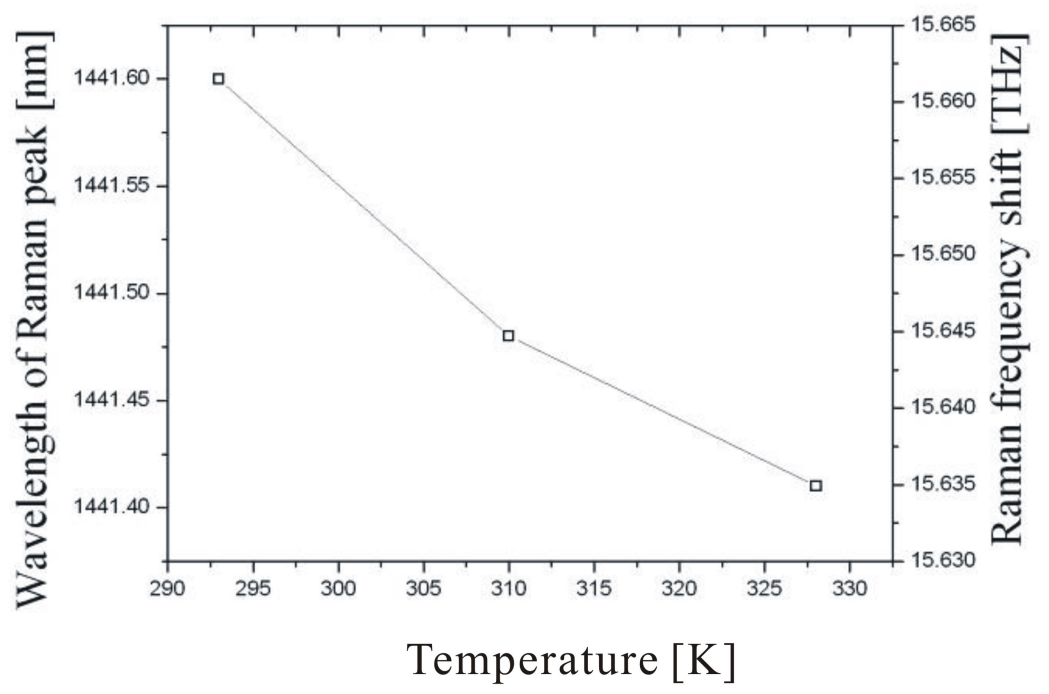

Fig.6 Raman frequency shift in silicon waveguide vs. temperature

From Fig.6, we see that with increasing temperature, the Raman frequency shift decreases. From the experiment we expect a temperature dependence of Raman shift of about $0.6 \mathrm{GHz} / \mathrm{K}$, which is nearly the same as described in [6]. They also reported that the Raman linewidth increases around $0.1 \mathrm{GHz} / \mathrm{K}$. But this has not been observed in our experiments, mainly due to the resolution limitation. The reason why Raman shift and linewidth are temperature dependent [7] is that rising in temperature results in an increase in the interatomic distance and a reduction of the phonon lifetime. The former leads to a decrease in the optical phonon frequency and correspondingly to an increase in the frequency of the Stokes component. The latter, which is interpreted as an increase in the damping coefficient in the context of a classical oscillator model, leads to broadening of the optical phonon linewidth.

The first order anti-Stokes Raman emission is also measured, shown in Fig. 7. The pink (It will be in black and white) line shows the first order anti-Stokes Raman emission, while the black line shows the first Stokes Raman emission with the same pump power. The ratio between the intensity of first anti-Stokes and first stokes is measured as around 1/12, which can be predicted by Eq. (2)

$\frac{I_{a s}}{I_{s}}=\exp \left(-\frac{h v_{\text {phonon }}}{k T}\right) \approx 0.08$ 


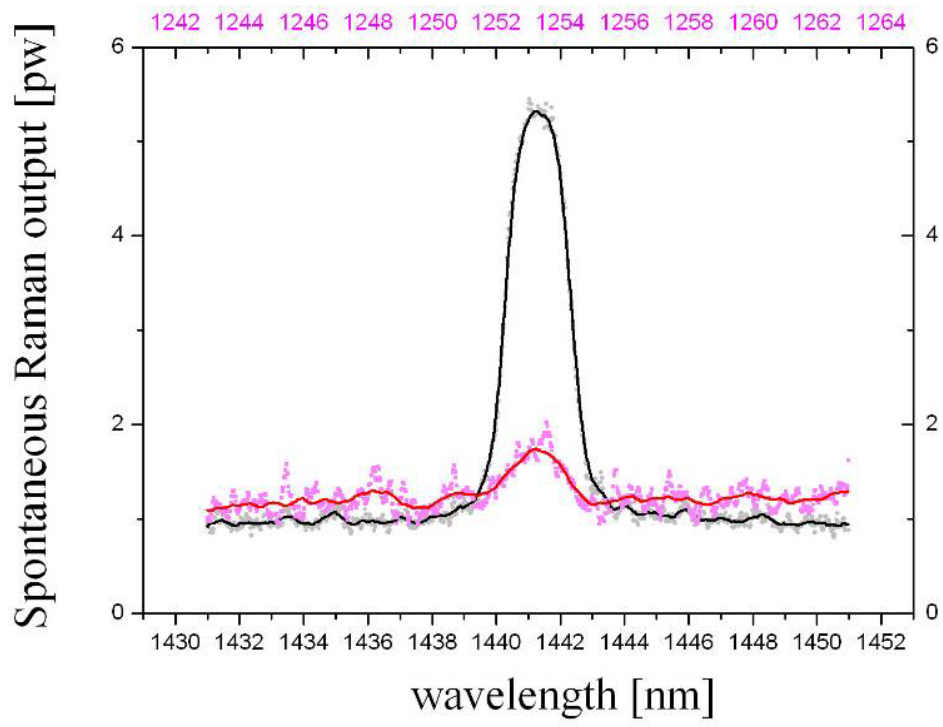

Fig. 7 Comparision of anti-Stokes and Stockes Raman emission in silicon waveguide

\section{STIMULATED RAMAN EMISSION IN PLANAR SILICON WAVEGUIDE}

At last we also measured the stimulated Raman amplification in such a planar silicon waveguide. The experimental setup is shown in Fig.8.

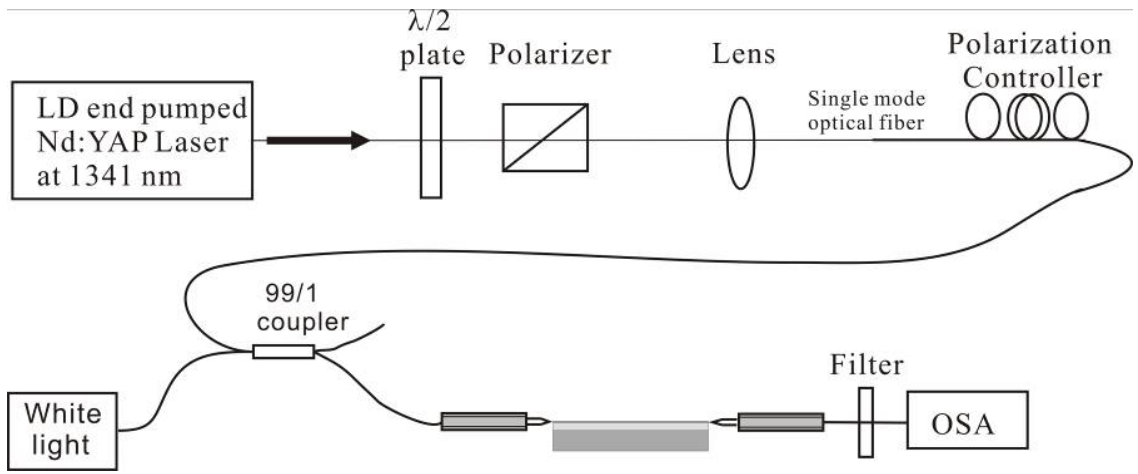

Fig.8 Experiment of stimulated Raman amplification in planar silicon waveguide

The $\mathrm{cw} 1341 \mathrm{~nm} \mathrm{Nd:YAP}$ laser is used as the pump source again. A white light source which is centered at around $1440 \mathrm{~nm}$ is used as the probe source. A 99/1 coupler is used to couple the pump and probe together. The tapered fiber helps to couple the pump and probe into the silicon waveguide. The filter is used to block the pump wavelength from Raman emission. At first, the OSA measured the output of white light source without pump power. Then the OSA measured the output of white light source with pump power. We subtract these two outputs, and could obtain the amplification curve. Fig. 
9 shows the experimental results. The blue $(b \& w)$ curve is measured spontaneous Raman spectrum, while the black curve is the measured Raman amplification. The resolution of OSA is used as $2 \mathrm{~nm}$, that is to say the true amplification factor is around 2 times larger than measured by OSA considering the linewidth of spontaneous Raman emission is around $1 \mathrm{~nm}$. We could see from the figure that the measured amplification curve fits well with the measured spontaneous Raman spectrum.

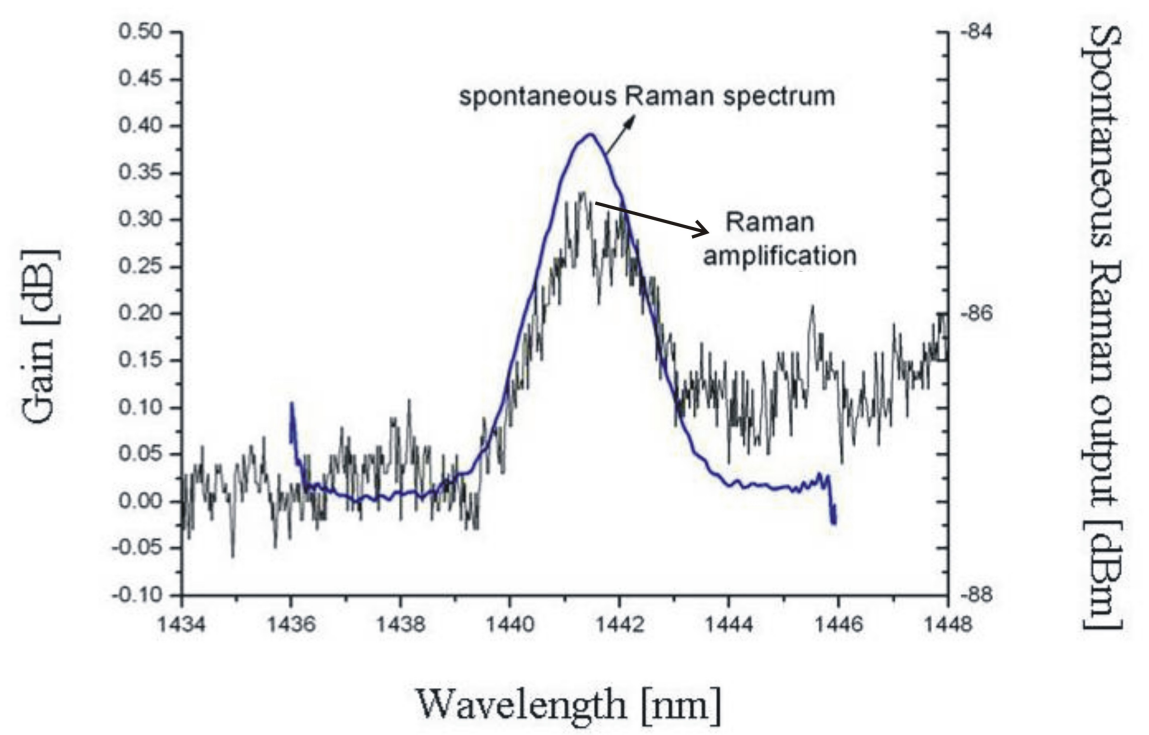

Fig. 9 Measured Raman amplification curve and spontaneous Raman spectrum curve

Fig. 10 shows the measured stimulated Raman gain dependent on the pump power at fiber output. With around $350 \mathrm{~mW}$ pump power, maximum of around $0.6 \mathrm{~dB}$ gain is obtained. The stimulated Raman gain coefficient is estimated to $35 \mathrm{~cm} / \mathrm{GW}$.

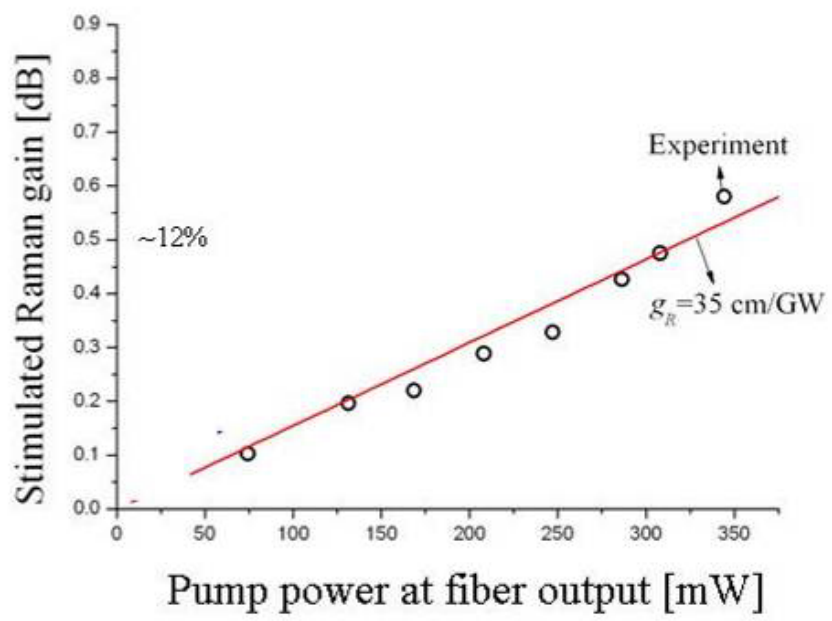

Fig. 10 Measured stimulated Raman gain dependent on pump power at fiber output 


\section{CONCLUSION}

In this paper, we measured both spontaneous Raman output and stimulated Raman amplification of a silicon on insulator (SOI) waveguide with $2 \mu \mathrm{m}$ width, $220 \mathrm{~nm}$ height and $2 \mathrm{~cm}$ length. With $1341 \mathrm{~nm}$ pump, the first stokes is measured at $1441.6 \mathrm{~nm}$. The temperature dependence of Raman shift is measured as about $0.6 \mathrm{GHz} / \mathrm{K}$. The first anti-stokes which centered at $1253 \mathrm{~nm}$ is also measured. At last we obtained the stimulated Raman amplification of such a small silicon waveguide. Maximum gain of around $0.6 \mathrm{~dB}$ is obtained at $350 \mathrm{~mW}$ pump.

\section{REFERENCES:}

[1] P.A. Temple, C.E. Hathaway, "Multiphonon Raman spectrum of silicon", Phys. Rev. B, 7, 3685 (1973)

[2] R. Claps, D. Dimitropoulos, Y. Han and B. Jalali, "Observation of Raman emission in silicon waveguides at $1.54 \mu \mathrm{m}$ " Opt. Express, 10, 1350, (2002)

[3] S. Meister; A. Al-Saadi; B. A. Franke; S. Mahdi; B. Kuhlow; K. Voigt; B. Tillack; H.H. Richter; L. Zimmermann; V. Ksianzou; S.K. Schrader; and H.J. Eichler, "Photonic crystal microcavities in SOI waveguides produced in a CMOS environment", SPIE proceeding, 7606, 760616, (2010)

[4] M. Krause, H. Renner and E. Brinkmeyer, "Analysis of Raman lasing characteristics in silicon-on-insulator waveguides”, Opt. Express, 12, 5703, (2004)

[5] http://www.efunda.com/materials/elements/TC_Table.cfm?Element_ID=Si

[6] T. K. Liang and H. K. Tsang, "Optical limiting and Raman amplification in silicon waveguide", in Optical Fiber Communication Conference and Exposition and The National Fiber Optic Engineers Conference, Technical Digest (CD), paper JWA15, Optical Society of America, (2005)

[7] W. Kim and D. Park, "Analysis of temperature effects on Raman silicon photonic devices", J. Opt. Soc. Korea, 12, 288, (2008) 